\title{
Penetration of a supersonic particle at the interface in a binary complex plasma
}

\author{
He Huang $\odot,{ }^{1}$ Mierk Schwabe $\odot,{ }^{2}$ Hubertus M. Thomas, ${ }^{2}$ Andrey M. Lipaev,,${ }^{3,4}$ and Cheng-Ran Du $\odot^{1,5, *}$ \\ ${ }^{1}$ College of Science, Donghua University, Shanghai 201620, People's Republic of China \\ ${ }^{2}$ Institut für Materialphysik im Weltraum, Deutsches Zentrum für Luft- und Raumfahrt (DLR), Weßling 82234, Germany \\ ${ }^{3}$ Joint Institute for High Temperature, Moscow 125412, Russia \\ ${ }^{4}$ Moscow Institute of Physics and Technology (MIPT), Dolgoprudny 141701, Russia \\ ${ }^{5}$ Member of Magnetic Confinement Fusion Research Centre, Ministry of Education, Shanghai 201620, People's Republic of China
}

(Received 15 March 2020; revised 23 November 2020; accepted 15 December 2020; published 8 January 2021)

\begin{abstract}
The penetration of a supersonic particle at the interface is studied in a binary complex plasma. Inspired by the experiments performed in the PK-3 Plus Laboratory on board the International Space Station, Langevin dynamics simulations were carried out. A Mach cone structure forms in the lateral wave behind the supersonic extra particle, where the kink of the cone flanks is observed at the interface. The propagation of the pulse-like perturbation along the interface is demonstrated by the evolution of the radial and axial velocity of the small particles in the vicinity of the interface. The decay of the pulse strength is determined by the friction, where the propagation distance can reach several interparticle distances for small damping rate. The dependence of the dynamics of the background particles in the vicinity of the interface on the penetration direction implies that the disparity of the mobility may be the cause of various interfacial effects.
\end{abstract}

DOI: 10.1103/PhysRevE.103.013205

\section{INTRODUCTION}

A complex plasma is a weakly ionized gas containing small solid particles [1,2]. The particles are highly charged by collecting ions and electrons. Using video microscopy, localized structures and dynamics can be directly recorded in the experiments. Various phenomena such as formation of crystal lattice $[3,4]$, wave phenomena [5-10], and instabilities [11,12] can be studied in complex plasmas. A binary complex plasma contains two types of microparticles of different sizes, which can either be mixed $[13,14]$ or form a phase separated system [15-17]. It was discovered that phase separation can still occur due to the difference in force balance for microparticles of different sizes under microgravity conditions despite the criteria of spinodal decomposition not being fulfilled $[18,19]$. An interface between separated phases emerges and various interfacial phenomena are investigated [20,21].

Recently, wakes excited by a moving disturbance in complex plasmas have attracted much attention [22-27] since the first theoretical predictions [28,29]. The disturbance can be imposed by either a laser beam [30,31] or extra particles [32-34]. If the disturbance moves faster than the sound speed in the complex plasma, the wakes exhibit a V-shaped structure in the two-dimensional (2D) case [35,36] and a conical structure in the three-dimensional (3D) case [32,33,37], known as Mach cone. In the ground laboratory, the particles are levitated in the (pre)sheath and form a 2D plasma crystal in the case of strong coupling conditions. The extra particles can travel either above or below the particle layer, exciting a repulsive or attractive Mach cone, respectively $[35,38]$. Under

\footnotetext{
*chengran.du@dhu.edu.cn
}

microgravity conditions, the particles form a relatively homogeneous 3D complex plasma. The penetration of the extra particles results in a moving disturbance inside the particle cloud, generating a 3D Mach cone if moving faster than the speed of sound $[32,37,39,40]$.

In this paper, we present a numerical simulation to study the evolution of a lateral wake excited by a supersonic extra particle at the interface, inspired by an experimental observation under microgravity conditions. In Sec. II, the experimental setup and the observations are introduced. In Sec. III, the method of the numerical simulations is described and the parameters of two series of simulations are listed. In Sec. IV, we analyze the numerical simulations in three aspects including the Mach cone behind the extra particle (Sec. IV A), the pulses propagating along the interface (Sec. IV B), and the closure of the cavity at the interface (Sec. IV C). The results are discussed in Sec. V. Finally, a conclusion is drawn in Sec. VI.

\section{EXPERIMENTS}

The experiments were performed in the PK-3 Plus Laboratory on board the International Space Station (ISS). The sketch of the discharge chamber is shown in Fig. 1 and the technical details can be found in the Ref. [41]. A neon plasma was produced by a capacitively coupled radiofrequency (rf) generator in push-pull mode at $13.56 \mathrm{MHz}$. The binary complex plasma was prepared by injecting two types of particles. The first type is melamine formaldehyde (MF) particles of a diameter of $3.42 \mu \mathrm{m}$ with a mass $m_{b}=3.3 \times 10^{-14} \mathrm{~kg}$, while the second type is $\mathrm{SiO}_{2}$ particles of a diameter of $1.55 \mu \mathrm{m}$ with a mass $m_{s}=3.6 \times 10^{-15} \mathrm{~kg}$. A void was generated in the center of the particle cloud due to the presence of the ion 


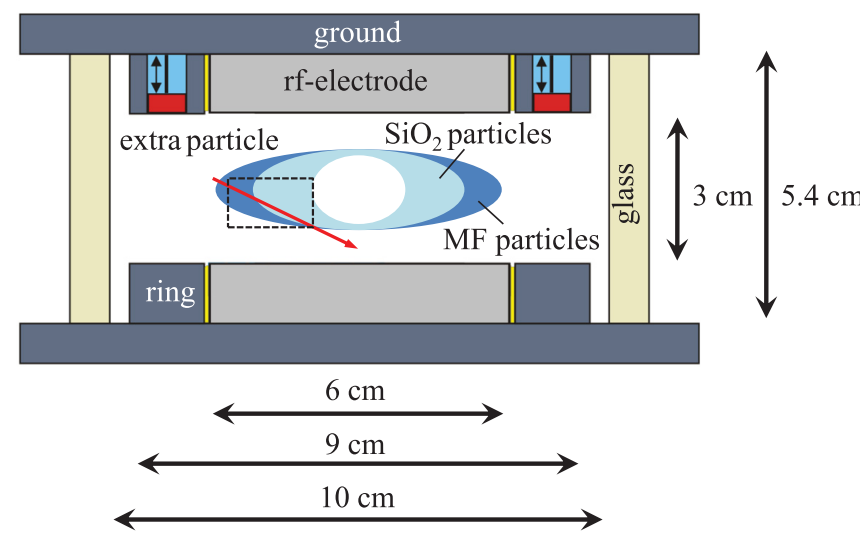

FIG. 1. The setup of PK-3 Plus on board the International Space Station. The red arrow represents the trajectory of the extra particle, penetrating the phase separated binary complex plasma. The region of interest is highlighted by a dashed rectangle.

flows, as shown in Fig. 1. In addition, agglomerated larger particles were present on the outside of the particle cloud. Using video microscopy [41], a cross section of particle cloud (illuminated by a laser sheet) was recorded at a rate of 50 frames per second (fps). The gas pressure was set at $20 \mathrm{~Pa}$, and the discharge voltage was set at $20 \mathrm{~V}$.

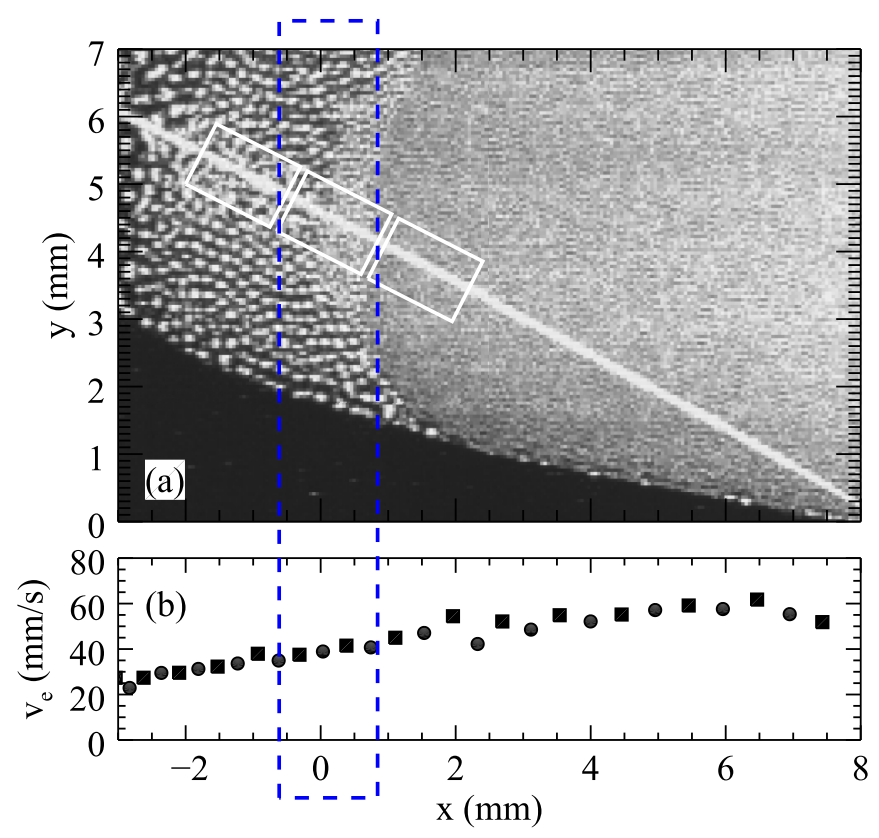

FIG. 2. Snapshot of the penetration of an extra particle across the interface $(x \sim 0 \mathrm{~mm})$ of a binary complex plasma in the experiment (a) and velocity of the extra particle (b). The area of the panel (a) corresponds to the dashed rectangle in Fig. 1. 15 consecutive images are overlaid, where the trajectory of the penetrating particle is shown as a straight white line. Three locations are highlighted by the white solid rectangles, corresponding to the insets in Fig. 3(a-c). In (b), the velocity is measured by tracking the positions of the extra particle (circles) or measuring the length of its elongated shape in each recorded frame (squares), see also the insets in Fig. 3(a-c). The blue dashed rectangle highlights the region of interface.
As we can see in Fig. 2, the particles of two types were phase-separated, mainly due to the difference of the ion drag force [19]. The small particles were confined on the right side, while the big particles were on the left side. Due to the low spatial resolution of the camera equipped on the space station, small particles cannot be identified individually and thus their number density remains unknown. The extra particle moved from the left to the right, leaving a straight trajectory across the interface. The driving force is still not fully understood [37]. It might be due to a rocket force acting on the extra particle [42].

We employed the PYTHON library AIRCV based on the SIFT feature detector algorithm [43] to track the penetrating particle. The velocity of the extra particle can be obtained using two methods. First, it can be calculated by comparing the tracked positions in two consecutive frames, as shown by the circles in Fig. 2(b). Second, the velocity can be directly obtained by measuring the length of the elongated extra particle in each frame, as shown by the squares in Fig. 2(b). It turns out that the measured velocities using two methods agree with each other. As result, the extra particle accelerated from $20 \mathrm{~mm} / \mathrm{s}$ to $60 \mathrm{~mm} / \mathrm{s}$, and the velocity near the interface was $\sim 40 \mathrm{~mm} / \mathrm{s}$.

Due to the strong Yukawa interaction, the extra particle repelled both small and big particles in its vicinity as it penetrated the particle cloud. As we see in the insets of Fig. 3(a-c), a cavity around the extra particle appeared in the experimental observations. Since the small particles have lower charge, the size of the cavity was smaller in the small particle cloud than that in the big particle cloud.

At the beginning of the penetration in the experiment, the velocity of the extra particle was relatively low and we did not observe a cone structure behind the extra particles. This implies that the velocity of the extra particle was smaller than the sound speed of the big particle cloud. The Mach cone structure of the lateral wake started to emerge as the extra particle crossed the interface. The structure became evident as its velocity reached $v_{e} \sim 60 \mathrm{~mm} / \mathrm{s}$, where the cone angle decreased dramatically, as shown in Fig. 4. The cone angle was directly measured as $\theta=25^{\circ}$. The sound speed of the small particle cloud can then be estimated as $c_{s} \sim 25 \mathrm{~mm} / \mathrm{s}$.

The penetration of the extra particle at the interface excited a pulse-like perturbation, propagating along the interface. For the experiment, we select three horizontal strips with range $x=-3$ to $8 \mathrm{~mm}$ at the vertical locations $y=5.2,5.35$, $5.5 \mathrm{~mm}$, corresponding to the radial distance $r=0.5,0.68$, $0.86 \mathrm{~mm}$ (perpendicular to the linear trajectory of the extra particle), respectively, and plot the periodgrams. As we see in Fig. 5(b,c), there is a bump at $t \sim 0 \mathrm{~s}$ at both $r \sim 0.5$ and $0.68 \mathrm{~mm}$, representing a pulse-like perturbation propagating along the interface. However, due to the low temporal and spatial resolution of the recorded video, one can neither conclude the propagation direction of this pulse nor calculate the velocity and magnitude of this perturbation from the analysis. This perturbation is not seen for $r<0.5 \mathrm{~mm}$ (not shown here) due to the presence of the cavity and vanishes at $r=0.86 \mathrm{~mm}$, as shown in Fig. 5(a), implying a short propagation distance.

However, due to the high density of the background particles, it is difficult to obtain the full details of individual particles in the cross section. In order to study the dynamics 

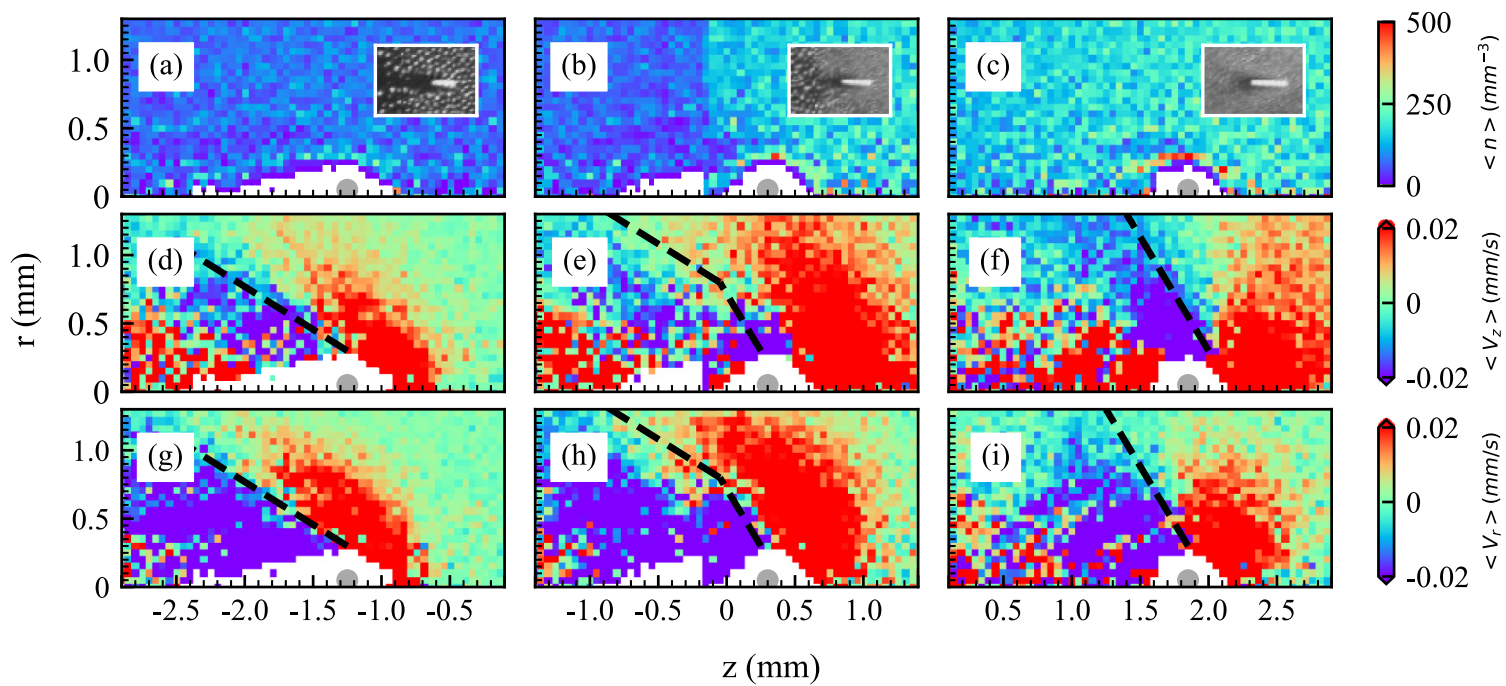

FIG. 3. Distribution of the density $(\mathrm{a}-\mathrm{c})$ and the velocity in $z$ direction $(\mathrm{d}-\mathrm{f})$ and in radial direction $(\mathrm{g}-\mathrm{i})$ of the background particles in a binary complex plasma in the Langevin dynamics simulation. The penetration velocity of the extra particle (shown as a grey semicircle) is set as $50 \mathrm{~mm} / \mathrm{s}$. The left, middle, and right panels correspond to the moment where the extra particle is in the big particle cloud, in the vicinity of the interface, and in the small particle cloud, respectively. For comparison, the experiment images at a similar moment are shown in the inset of $(\mathrm{a}-\mathrm{c})$, correspondingly. The cone structure is highlighted by dashed lines in $(\mathrm{d}-\mathrm{i})$.

of this phenomenon quantitatively, we performed Langevin dynamics simulations, where the positions and velocities of each particle can be acquired easily $[44,45]$, as we shall see next.

\section{SIMULATIONS}

In the Langevin dynamics simulations, the equation of motion including damping from the neutral gas is given as

$$
m_{i} \ddot{r}_{i}+m_{i} v_{i} \dot{r}_{i}=-\sum_{j \neq i} \nabla \phi_{i j}+F_{i d, i}+F_{c, i}+L_{i},
$$

where $r_{i}$ is the position of the particle $i, m_{i}$ is the mass, $v_{i}$ is the damping rate, and $L_{i}$ is the Langevin force. The Langevin force is defined by $\left\langle L_{i}(t)\right\rangle=0$ and $\left\langle L_{i}(t) L_{i}(t+\tau)\right\rangle=$

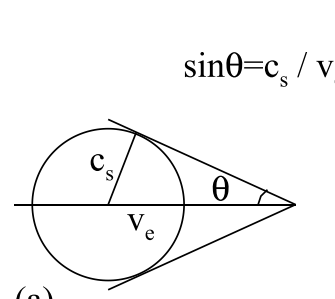

(a)

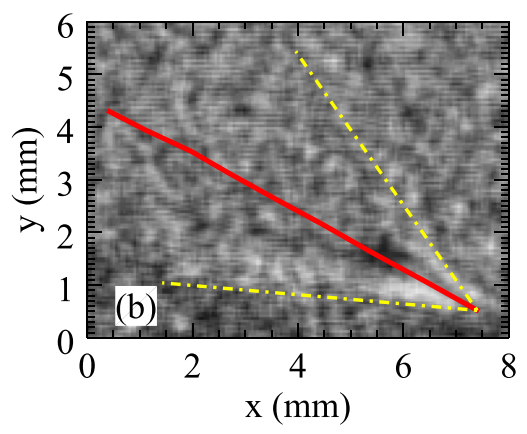

FIG. 4. Mach cone behind the supersonic particle. (a) Relation of the particle velocity $v_{e}$ and sound speed $c_{s}$ to the cone angle $\theta$. (b) The Mach cone structure observed behind the extra particle in the small particle cloud in the experiment. The image is produced by subtracting two consecutive frames to highlight the structure. The flanks of the cone are highlighted by yellow dashed-dotted lines and the trajectory of the extra particle is highlighted by the red-solid line.
$2 v_{i} m_{i} k_{B} T \delta(\tau) I$, where $T$ is the temperature of the heat bath, $\delta(\tau)$ is the delta function, and $I$ is the unit matrix.

In the simulation, we assumed that the particles interact with each other via the Yukawa potential,

$$
\phi_{i j}=\frac{Q_{i} Q_{j}}{4 \pi \epsilon_{0} r_{i j}} \exp \left(-\frac{r_{i j}}{\lambda}\right),
$$

where $\lambda$ is the Debye length, $Q_{i}$ is the charge of particle $i$, and $Q_{j}$ is the charge of a neighboring particle $j$, separated

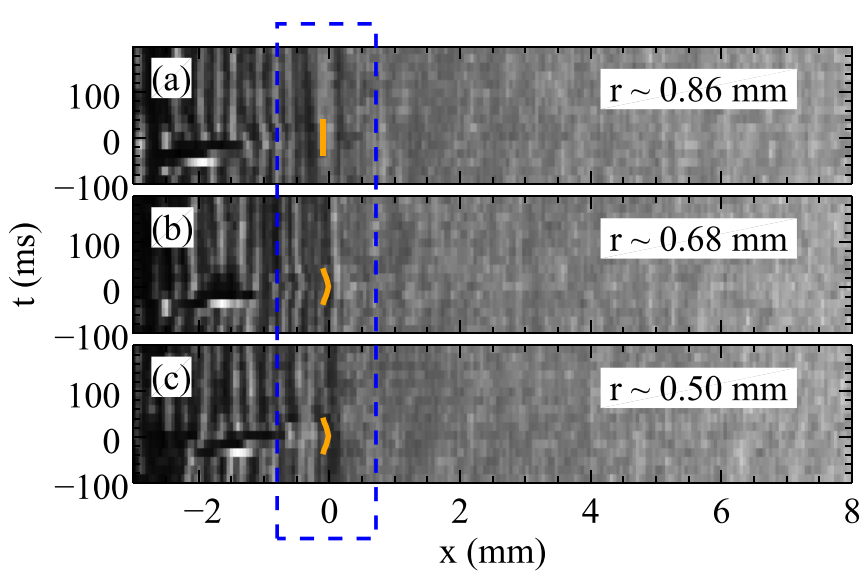

FIG. 5. The evolution of the pulse-like perturbation at the interface for radial locations $r \sim 0.5 \mathrm{~mm}$ (a), $r \sim 0.68 \mathrm{~mm}$ (b), and $r \sim 0.86 \mathrm{~mm}$ (c) in the experiment. The bump highlighted by the orange arc represents the axial motion of big particles in (b,c), excited by the penetration of extra particle at $r=0 \mathrm{~mm}$. Since the temporal resolution $(20 \mathrm{~ms})$ of the experiment is much larger than the characteristic time scale of the propagation of the pulse $\sim 10 \mathrm{~ms}$, one sees the pulse for both $r \sim 0.5 \mathrm{~mm}$ (a) and $r \sim 0.68 \mathrm{~mm}$ (b) at $t \approx 0 \mathrm{~ms}$. The axial perturbation vanishes for $r \sim 0.86 \mathrm{~mm}$ (c). The blue dashed rectangle highlights the region of interface and $t=0 \mathrm{~ms}$ represents the moment that the extra particle reaches the interface. 
TABLE I. Parameters in the Langevin dynamics simulation including mass $m_{b, s}$ and damping rate $v_{b, s}$ for big and small particles. Two series of simulations were performed. In series I, the damping rates corresponding to three neutral gas pressures $P=20,15,7.5 \mathrm{~Pa}$ were used, while in series II, the mass of small particles was adjusted. Here, we assume that the small particles are made of materials of smaller mass density, while their radius keeps constant.

\begin{tabular}{|c|c|c|c|c|c|}
\hline series & $\begin{array}{c}m_{b} \\
{[\mathrm{~kg}]}\end{array}$ & $\begin{array}{c}m_{s} \\
{[\mathrm{~kg}]}\end{array}$ & $\begin{array}{c}v_{b} \\
{\left[\mathrm{~s}^{-1}\right]}\end{array}$ & $\begin{array}{c}v_{s} \\
{\left[\mathrm{~s}^{-1}\right]}\end{array}$ & $\begin{array}{c}v_{e} \\
{[\mathrm{~mm} / \mathrm{s}]}\end{array}$ \\
\hline \multirow{3}{*}{ I } & $3.3 \times 10^{-14}$ & $3.6 \times 10^{-15}$ & 50.7 & 91.3 & 50 \\
\hline & $3.3 \times 10^{-14}$ & $3.6 \times 10^{-15}$ & 39.6 & 71.0 & 50 \\
\hline & $3.3 \times 10^{-14}$ & $3.6 \times 10^{-15}$ & 19.0 & 34.0 & 50 \\
\hline \multirow{3}{*}{ II } & $3.3 \times 10^{-14}$ & $3.6 \times 10^{-15}$ & 50.7 & 91.3 & $-110,-90, \ldots, 110$ \\
\hline & $3.3 \times 10^{-14}$ & $2.7 \times 10^{-15}$ & 50.7 & 91.3 & $-110,-90, \ldots, 110$ \\
\hline & $3.3 \times 10^{-14}$ & $1.8 \times 10^{-15}$ & 50.7 & 91.3 & $-110,-90, \ldots, 110$ \\
\hline
\end{tabular}

by interparticle distance $r_{i j}$. The particle cloud was confined by the ion drag force $F_{i d, i}$ directed in the negative $z$ direction, and the confinement force $F_{c, i}\left(=-\nabla \Phi Q_{i}\right)$ resulting from the confinement potential $\Phi$. The ion drag force for small particles was set as $F_{i d, s}=18 \mathrm{fN}$, while for big particle it was $F_{i d, b}=63 \mathrm{fN}$. The confinement potential was assumed to be parabolic, i.e., $\Phi=1 / 2 C z^{2}$, with a constant $C=10^{4} \mathrm{~V} / \mathrm{m}^{2}$. The charge for small and big particles were set as $Q_{s}=2700 \mathrm{e}$ and $Q_{b}=6000 \mathrm{e}$. The Debye length was $\lambda=100 \mu \mathrm{m}$. As result, the particle cloud was phase separated with the small particles located to the right of the big particles, as we see in Fig. 2. The exact location of the interface (marked by $z=0$ ) can be clearly identified as the sudden change of the particle number density in Fig. 2(b). In addition, the charge of the extra particle was set $Q_{e}=30000$ e.

However, one should be aware that the models applied in the simulation was rather simplified, so that it cannot fully mimic the conditions in the experiments, see Sec. $V$ for detailed discussions. Therefore, we selected and expanded the simulation parameters such that the phenomena induced by the extra particle can be clearly visible and easily analyzed to avoid ambiguity. A full correspondence between the simulation and experiment is beyond the scope of this work. The simulations were performed with 4000 small particles and 1500 big particles, using LAMMPS in NVE ensemble [46]. The rest of the parameters were set as in Table I [20,41,47]. Note that the simulation was performed in the Cartesian coordinate system, while the analysis was performed in cylindrical coordinates, considering the symmetry of the system.

\section{RESULTS}

\section{A. Mach cone behind the extra particle}

Despite the fact that the extra particle accelerated along its trajectory in the experiment, for simplicity we set the velocity of the extra particle as constant in the simulation to discard the effect caused by the change of the velocity. To focus on the Mach cone excited by the extra particle and its deformation at the interface, unlike in the experiments, we select the penetration velocity as $v_{e}=50 \mathrm{~mm} / \mathrm{s}$ so that it exceeds the sound speed in both small and big particle clouds in the simulations.

The density distribution in the simulation is shown in Fig. 3(a-c). Particle-free cavities are clearly seen around the extra particle, which are caused by its repulsion on the surrounding particles [26]. The cavity in the big particle cloud has a more elongated shape than that in the small particle cloud. However, it is truncated into two separated parts at the interface. This remarkable feature is caused by the fact that the small particles are much more mobile than the big particles so that they fill in the cavity at the interface before the big particles in the rear do.

The axial and the radial velocity distribution of the background particles are shown in Fig. 3(d-f) and 3(g-i), respectively. Clearly, the sound speed in the big particle cloud is smaller than that in the small particle cloud, which results in a smaller angle of the Mach cone. The flank of the cone is kinked at the interface, highlighted by the dashed lines in Fig. 3(e,h). We measure the cone angle in the small and big particle cloud as $\theta=57^{\circ}$ and $\theta=31^{\circ}$, respectively. This gives us the sound speed of small and big particle cloud as $42 \mathrm{~mm} / \mathrm{s}$ and $26 \mathrm{~mm} / \mathrm{s}$, accordingly. The sound speed of the small particle cloud in the simulation is larger than that in the experiment.

\section{B. Pulses along the interface}

In light of the experimental observations, we select all small particles within $50 \mu \mathrm{m}$ from the interface in the simulation and obtain the evolution of the axial and radial velocity. For better resolution, the simulation was repeated 20 times with different initial conditions, and the results were averaged. The results are shown in the periodgram in Fig. 6. The generation of the cavity at the interface excited the first pulse-like perturbation propagating outwards, highlighted by a red ellipse in the periodgram of $v_{r}$ in Fig. 6(a). The average radial velocity $v_{r}$ of the small particles in the pulse decreased from $10 \mathrm{~mm} / \mathrm{s}$ at the edge of the cavity to zero as the pulse reached $r \sim 1 \mathrm{~mm}$ within $15 \mathrm{~ms}$ before it vanished. ${ }^{1}$ As the extra particle left the interface, the cavity started shrinking instantaneously. The small particles refilled the cavity as shown by the blue strip highlighted by the blue ellipse in Fig. 6(a). As soon as the cavity was closed, a third pulse was excited, propagating along the interface outwards, highlighted by the green ellipse. At a neutral gas pressure of $20 \mathrm{~Pa}$, the last pulse

\footnotetext{
${ }^{1}$ Note that the time scale of this interfacial phenomenon is much faster than the temporal resolution provided by the equipment on the ISS.
} 

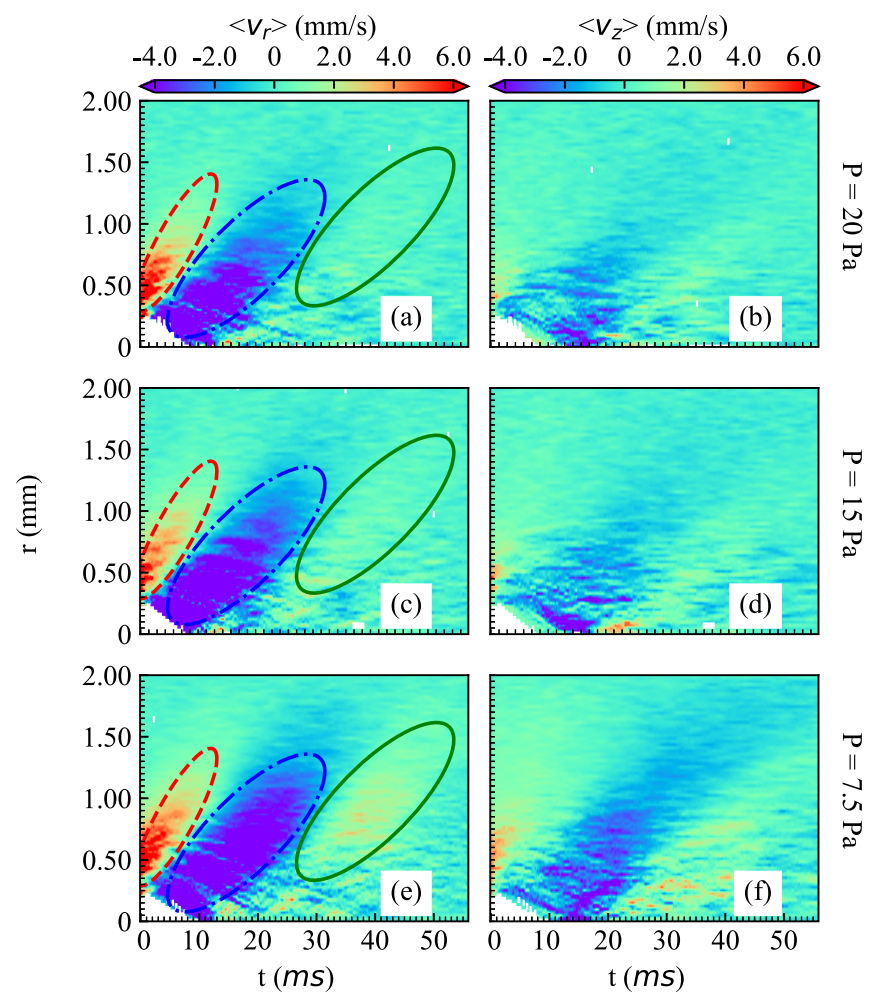

FIG. 6. The propagation of the pulse-like perturbation along the interface in terms of the radial velocity $(a, c, e)$ and axial velocity $(\mathrm{b}, \mathrm{d}, \mathrm{f})$ of the small particles in the simulations. The three selected pressures are $P=20,15,7.5 \mathrm{~Pa}$, corresponding to the damping rates of big particles $v_{b}=50.7,39.6,19.0 \mathrm{~s}^{-1}$ and small particles $v_{s}=91.3,71.0,34.0 \mathrm{~s}^{-1}$, respectively. The cavity is left blank. The first and third pulse with positive radial velocity are highlighted by the red and green ellipses, respectively, while the second pulse with negative radial velocity is highlighted by the blue ellipse.

was rather weak so that it propagated only about $0.5 \mathrm{~mm}$, mainly due to the damping. This result qualitatively agrees with the experimental observations though more details are revealed via the simulations.

In order to study the dependence of the propagation of such pulses along the interface on the damping, we ran the simulation with another two lower pressures. ${ }^{2}$ As we can see in Fig. 6(a-d), for a pressure of $15 \mathrm{~Pa}$, the propagation distance is slightly larger than that for $20 \mathrm{~Pa}$. The average radial and axial velocities of small particles in the pulses are comparable. As we further decrease the pressure to $7.5 \mathrm{~Pa}$, one sees clearly that the perturbation, particularly in the third pulse (marked by the green ellipse), propagates longer and further, up to a distance greater than $1.5 \mathrm{~mm}$, which is almost six times of the interparticle distance, until the average axial velocity $v_{r}$ drops to zero, as shown in Fig. 6(e).

This trend is also clearly demonstrated by the comparison of the radial velocity for three pressures in Fig. 7. For the

\footnotetext{
${ }^{2}$ It is challenging to perform the experiments at these low pressures since the two-stream instability may be triggered. With strong selfexcited waves, the perturbation along the interface can no longer be observed [20].
}

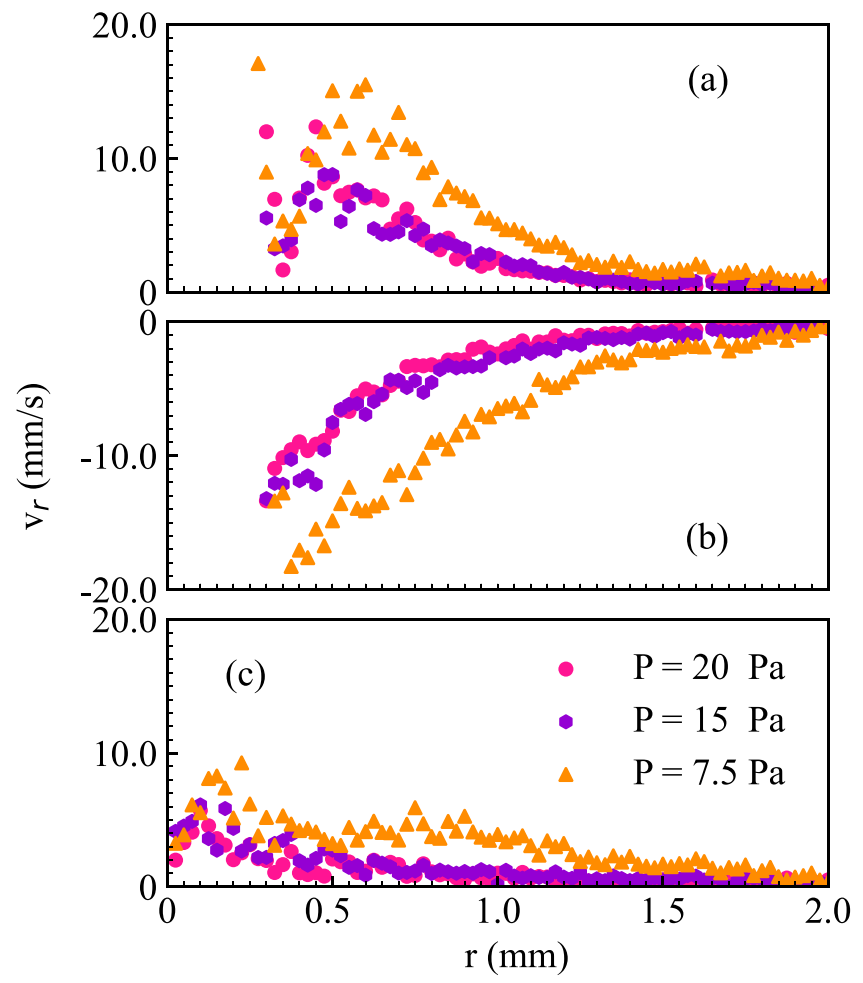

FIG. 7. Comparison of the maximal radial velocity in the first (a), second (b), and third (c) pulse propagating along the interface, corresponding to the area highlighted by the red, blue, and green ellipses in Fig. 6. The velocities for pressures $P=20,15,7.5 \mathrm{~Pa}$ are represented by pink, purple, yellow symbols, respectively.

pressure $P=20 \mathrm{~Pa}$ and $P=15 \mathrm{~Pa}$, the magnitude of the radial velocity in all three pulses are comparable. For much lower damping rate, the magnitude of $v_{r}$ is greater. However, this velocity decays to zero at the distance of $r \sim 1.5 \mathrm{~mm}$ for all three cases. The pulse-like perturbation indeed propagates like an interfacial wave excited by the penetration of the extra particle at the interface.

\section{Cavity closure by small particles}

It is interesting to study the dependence of the reaction of the background particles in the vicinity of the interface on the penetration direction and speed. A series of simulations were performed, where we varied the speed and direction of the extra particle. We define the penetration depth $\zeta$ as the maximal distance of the small particles entering the large particle cloud from the interface. A positive speed $v_{e}>0$ means that the extra particle moves from the cloud of big particles into that of small particles. As we depicted in Fig. 8(a), for positive penetration speeds, the small particles barely crossed the interface and intruded into the territory of the big particles. In contrast, for negative speeds, the small particles followed the extra particle, filled the cavity, and intruded into the territory of the big particles, as shown in Fig. 8(b).

This feature can also be revealed quantitatively in Fig. 9. For positive penetrating velocity, around two small particles on average moved across the interface. For negative penetrating velocity, the average number of small particles across the 


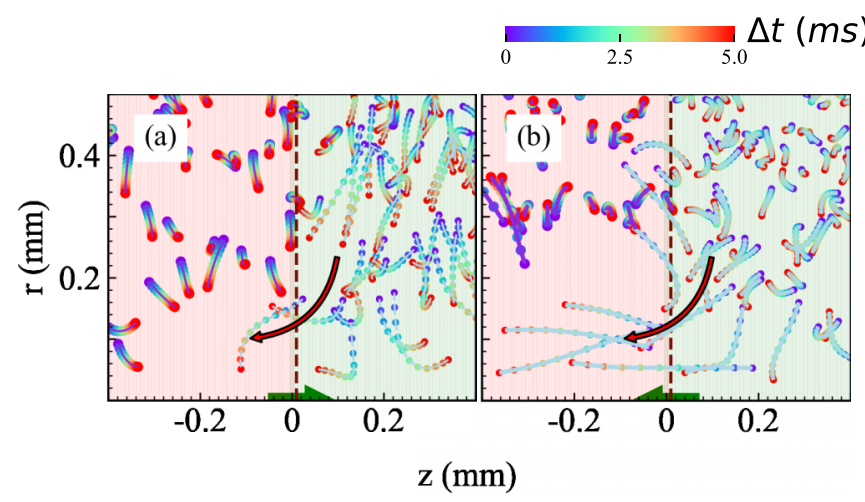

FIG. 8. Trajectories of background particles for two cases where the penetration directions of the extra particle are opposite. The green arrows represent the penetration direction of the extra particles. The penetration velocity is $v_{e}=30 \mathrm{~mm} / \mathrm{s}$ for (a) and $v_{e}=-30 \mathrm{~mm} / \mathrm{s}$ for (b). The colored curves represent the trajectories of particles at different times $\Delta t$, counting from the moment that the extra particle crossed the interface with a distance of $0.5 \mathrm{~mm}$. Thicker lines represent big particles while the thinner lines represent small particles. The red-dashed line is the position of the interface.

interface ranged from four to nine, depending on the magnitude of the velocity $v_{e}$.

As to the penetrating depth, at $v_{e} \approx-30 \mathrm{~mm} / \mathrm{s}$, the small particles reached the deepest depth as shown in Fig. 10. However, when the speed of the penetrating particles became even faster, the depth decreased and finally reached saturation. This is caused by the differences of the particles on the two sides of the interface, in terms of the mass, damping rate, as well as the strength of the interaction. These factors lead to the disparity of the particle mobility so that the small particles react with greater magnitude than big particles to the disturbances in the stimulations.

Generally speaking, particles with smaller mass react more promptly to the applied forces due to their small inertia. As the mobility of particles is closely related to the particle mass, we further study the dependence of the cavity closure on the mass of the small particles. Here, we assume that the small particles

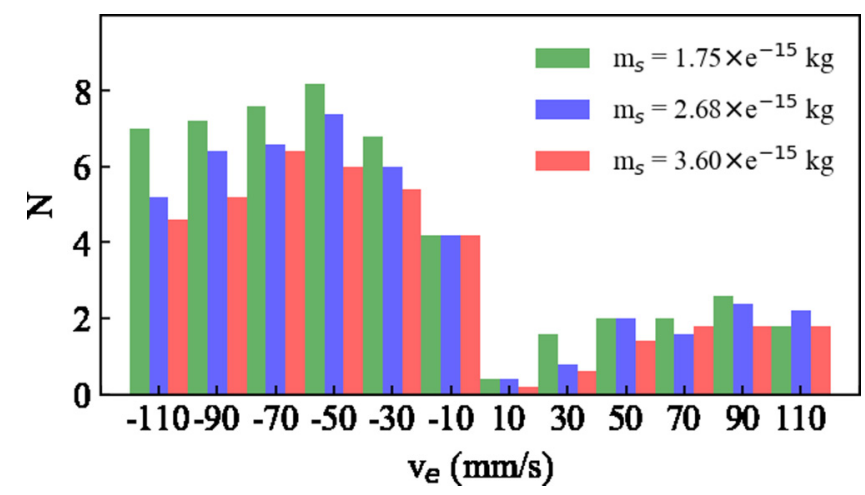

FIG. 9. Dependence of the number of small particles $N$, which refill the cavity and move across the interface, on the penetration velocity $v_{e}$ of the extra particle. Three types of small particles are considered, whose mass are $m_{s}=3.60 \times 10^{-15} \mathrm{~kg}$ (red bars), $m_{s}=$ $2.68 \times 10^{-15} \mathrm{~kg}$ (blue bars), and $m_{s}=1.75 \times 10^{-15} \mathrm{~kg}$ (green bars)

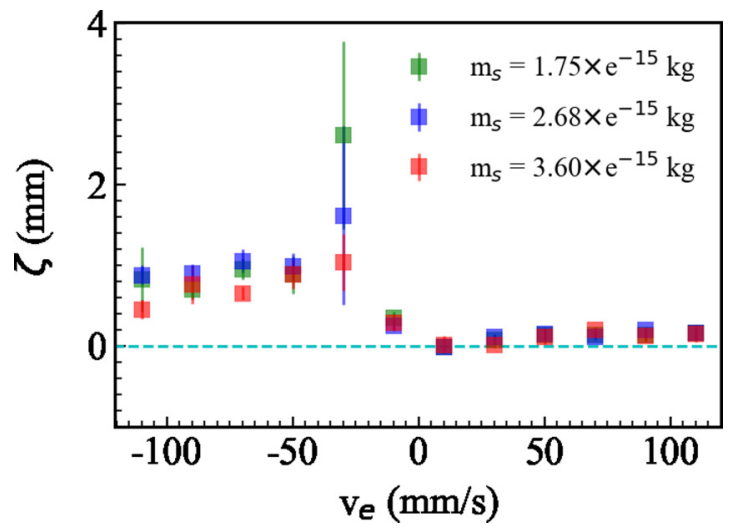

FIG. 10. The dependence of the maximal penetration depth $\zeta$ of small particles on the speed of the extra particle $v_{e}$, where the interface position is marked by a dashed line. Similar to Fig. 9, three types of small particles are considered whose masses are $m_{s}=3.60 \times 10^{-15} \mathrm{~kg}$ (red symbols), $m_{s}=2.68 \times 10^{-15} \mathrm{~kg}$ (blue symbols), and $m_{s}=1.75 \times 10^{-15} \mathrm{~kg}$ (green symbols). The errors signify the standard deviation across ten simulations with the same parameters.

are made of materials of smaller mass density. Meanwhile, the particle diameter and thus the surface charge do not change. As we see in Fig. 9, the number of the small particles $N$ across the interface depends on the disparity of the mass. For the positive $v_{e}$, the number of small particles across the interface does not make a difference for the three pairs of masses. However, for the negative $v_{e}$, the larger the disparity of the masses of the two types of particles, the more small particles move to the other side of the interface. Similarly, as we see in the Fig. 10, the maximal penetration depth strongly depends on the mass of the small particles. For the small particle of $1.7 \times 10^{-9} \mathrm{~kg}$, the maximal penetrating depth $\zeta$ exceeds $2.5 \mathrm{~mm}$ at $v_{e}=-30 \mathrm{~mm} / \mathrm{s}$. As the mass disparity of the two particle types increases, the difference of dynamics of particles on the two sides of the interface leads to an explicit phenomenon, which can be visualized macroscopically.

\section{DISCUSSION}

It is noteworthy that the situation in the experiments is more complicated than in the simulations. The plasma parameters in the discharge chamber are not uniform [48]. Therefore, the particle charge and the ion drag force for small and big particles are rarely constant. Furthermore, due to the presence of the ion flow, implied by the presence of the void, the interactions between particles may not be isotropic [49] and have a more complicated form than the Yukawa interaction. All these may contribute to the deviation of the simulation results from the experimental observations. However, it is beyond the scope of this paper to fully simulate the experimental observations. With the simplified model applied in the Langevin dynamics simulations, the origin of the interfacial phenomena induced by the penetration of a supersonic extra particle can already be demonstrated.

In fact, the disparity of the particle mobility may be the essential cause of various interface effects such as reflections of the solitary waves. In Ref. [21], a dissipative solitary wave 
was excited by external electric field in the small particles in a phase-separated binary complex plasma. This solitary wave reached the interface, drove small particles to move forward. However, since the mass of big particles is larger than that of small particles, the small particles rebounded while momentum transfer and a reflection of the solitary wave was observed, only when the damping was low enough. In Ref. [50], nonlinear solitary waves were excited in a binary granular material. The transmission and reflection at a oblique interface depended also on the disparity of the mass of the two particle types.

\section{CONCLUSION}

In conclusion, we performed Langevin dynamics simulations to study the penetration of a supersonic particle at an interface in a binary complex plasma. A Mach cone structure was observed, where a kink emerged at the interface. By studying the dependence of the reaction of the background particles in the vicinity of the interface on the penetration direction and speed, we show that the disparity of the mobility (represented by mass) between the particles on both sides of the interface has a significant influence on the interfacial effects.

\section{ACKNOWLEDGMENTS}

The authors acknowledge support from the National Natural Science Foundation of China (NSFC), Grants No. 11975073 and No. 12035003. The PK-3 Plus project was funded by the space agency of the Deutsches Zentrum für Luft- und Raumfahrt e.V. with funds from the Federal Ministry for Economy and Technology according to a resolution of the Deutscher Bundestag under Grant No. 50WP1203. The authors acknowledge Roscosmos provided the PK-3 Plus laboratory launch and operation on board of ISS. We would like to thank V. Nosenko for carefully checking the manuscript.
[1] V. Fortov, A. Ivlev, S. Khrapak, A. Khrapak, and G. Morfill, Phys. Rep. 421, 1 (2005).

[2] G. E. Morfill and A. V. Ivlev, Rev. Mod. Phys. 81, 1353 (2009).

[3] J. H. Chu and Lin I, Phys. Rev. Lett. 72, 4009 (1994).

[4] H. Thomas, G. E. Morfill, V. Demmel, J. Goree, B. Feuerbacher, and D. Möhlmann, Phys. Rev. Lett. 73, 652 (1994).

[5] P.-C. Lin and Lin I, Phys. Rev. Lett. 120, 135004 (2018).

[6] M. Schwabe, M. Rubin-Zuzic, S. Zhdanov, H. M. Thomas, and G. E. Morfill, Phys. Rev. Lett. 99, 095002 (2007).

[7] R. L. Merlino, J. Plasma Phys. 80, 773 (2014).

[8] P. Bandyopadhyay, G. Prasad, A. Sen, and P. K. Kaw, Phys. Rev. Lett. 101, 065006 (2008).

[9] E. Thomas, Phys. Plasmas 13, 042107 (2006).

[10] J. D. Williams, Phys. Rev. E 89, 023105 (2014).

[11] M. Schwabe, M. Rubin-Zuzic, S. Zhdanov, A. V. Ivlev, H. M. Thomas, and G. E. Morfill, Phys. Rev. Lett. 102, 255005 (2009).

[12] L. Couëdel, V. Nosenko, A. V. Ivlev, S. K. Zhdanov, H. M. Thomas, and G. E. Morfill, Phys. Rev. Lett. 104, 195001 (2010).

[13] H. Huang, A. V. Ivlev, V. Nosenko, Y.-F. Lin, and C.-R. Du, Phys. Plasmas 26, 013702 (2019).

[14] C.-R. Du, V. Nosenko, H. M. Thomas, Y.-F. Lin, G. E. Morfill, and A. V. Ivlev, Phys. Rev. Lett. 123, 185002 (2019).

[15] A. Wysocki, C. Räth, A. V. Ivlev, K. R. Sütterlin, H. M. Thomas, S. Khrapak, S. Zhdanov, V. E. Fortov, A. M. Lipaev, V. I. Molotkov et al., Phys. Rev. Lett. 105, 045001 (2010).

[16] K. Jiang, L.-J. Hou, A. V. Ivlev, Y.-F. Li, C.-R. Du et al., Europhys. Lett. 93, 55001 (2011).

[17] C.-R. Du, K. R. Sütterlin, K. Jiang, C. Räth, A. V. Ivlev, S. Khrapak, M. Schwabe, H. M. Thomas, V. E. Fortov, A. M. Lipaev et al., New J. Phys. 14, 073058 (2012).

[18] K. R. Sütterlin, A. Wysocki, A. V. Ivlev, C. Räth, H. M. Thomas, M. Rubin-Zuzic, W. J. Goedheer, V. E. Fortov, A. M. Lipaev, V. I. Molotkov et al., Phys. Rev. Lett. 102, 085003 (2009).

[19] C. Killer, T. Bockwoldt, S. Schütt, M. Himpel, A. Melzer, and A. Piel, Phys. Rev. Lett. 116, 115002 (2016).
[20] L. Yang, M. Schwabe, S. Zhdanov, H. M. Thomas, A. M. Lipaev, V. I. Molotkov, V. E. Fortov, J. Zhang, and C.-R. Du, Europhys. Lett. 117, 25001 (2017).

[21] W. Sun, M. Schwabe, H. M. Thomas, A. M. Lipaev, V. I. Molotkov, V. E. Fortov, Y. Feng, Y.-F. Lin, J. Zhang, Y. Guo et al., Europhys. Lett. 122, 55001 (2018).

[22] D. H. E. Dubin, Phys. Plasmas 7, 3895 (2000).

[23] S. Zhdanov, C.-R. Du, M. Schwabe, V. Nosenko, H. M. Thomas, and G. E. Morfill, Europhys. Lett. 114, 55002 (2016).

[24] V. Nosenko, S. Zhdanov, and G. Morfill, Phys. Rev. Lett. 99, 025002 (2007).

[25] C.-R. Du, V. Nosenko, S. Zhdanov, H. M. Thomas, and G. E. Morfill, Phys. Rev. E 89, 021101(R) (2014).

[26] S. Khrapak, P. Huber, H. Thomas, V. Naumkin, V. Molotkov, and A. Lipaev, Phys. Rev. E 99, 053210 (2019).

[27] D. I. Zhukhovitskii, V. E. Fortov, V. I. Molotkov, A. M. Lipaev, V. N. Naumkin, H. M. Thomas, A. V. Ivlev, M. Schwabe, and G. E. Morfill, Phys. Plasmas 22, 023701 (2015).

[28] O. Havnes, T. Aslaksen, T. W. Hartquist, F. Li, F. Melandso, G. E. Morfill, and T. Nitter, J. Geophys. Res.: Space Phys. 100, 1731 (1995).

[29] O. Havnes, F. Li, F. Melandso, T. Aslaksen, T. W. Hartquist, G. E. Morfill, T. Nitter, and V. Tsytovich, J. Vac. Sci. Technol. A 14, 525 (1996).

[30] A. Melzer, S. Nunomura, D. Samsonov, Z. W. Ma, and J. Goree, Phys. Rev. E 62, 4162 (2000).

[31] V. Nosenko, J. Goree, Z. W. Ma, and A. Piel, Phys. Rev. Lett. 88, 135001 (2002).

[32] K. Jiang, V. Nosenko, Y. F. Li, M. Schwabe, U. Konopka, A. V. Ivlev, V. E. Fortov, V. I. Molotkov, A. M. Lipaev, O. F. Petrov et al., Europhys. Lett. 85, 45002 (2009).

[33] D. Caliebe, O. Arp, and A. Piel, Phys. Plasmas 18, 073702 (2011).

[34] M. Schwabe, S. Zhdanov, T. Hagl, P. Huber, A. M. Lipaev, V. I. Molotkov, V. N. Naumkin, M. Rubin-Zuzic, P. V. Vinogradov, E. Zaehringer et al., New J. Phys. 19, 103019 (2017). 
[35] D. Samsonov, J. Goree, Z. W. Ma, A. Bhattacharjee, H. M. Thomas, and G. E. Morfill, Phys. Rev. Lett. 83, 3649 (1999).

[36] D. Samsonov, J. Goree, H. M. Thomas, and G. E. Morfill, Phys. Rev. E 61, 5557 (2000).

[37] M. Schwabe, K. Jiang, S. Zhdanov, T. Hagl, P. Huber, A. V. Ivlev, A. M. Lipaev, V. I. Molotkov, V. N. Naumkin, K. R. Sütterlin et al., Europhys. Lett. 96, 55001 (2011).

[38] C.-R. Du, V. Nosenko, S. Zhdanov, H. M. Thomas, and G. E. Morfill, Europhys. Lett. 99, 55001 (2012).

[39] D. I. Zhukhovitskii, V. E. Fortov, V. I. Molotkov, A. M. Lipaev, V. N. Naumkin, H. M. Thomas, A. V. Ivlev, M. Schwabe, and G. E. Morfill, Phys. Rev. E 86, 016401 (2012).

[40] E. Zaehringer, M. Schwabe, S. Zhdanov, D. P. Mohr, C. A. Knapek, P. Huber, I. L. Semenov, and H. M. Thomas, Phys. Plasmas 25, 033703 (2018).

[41] H. M. Thomas, G. E. Morfill, V. E. Fortov, A. V. Ivlev, V. I. Molotkov, A. M. Lipaev, T. Hagl, H. Rothermel, S. A. Khrapak, R. K. Sütterlin et al., New J. Phys. 10, 033036 (2008).
[42] V. Nosenko, A. V. Ivlev, and G. E. Morfill, Phys. Plasmas 17, 123705 (2010)

[43] D. G. Lowe, International Journal of Computer Vision 60, 91 (2004).

[44] L. J. Hou, Z. L. Miskovic, A. Piel, and P. K. Shukla, Phys. Plasmas 16, 053705 (2009).

[45] M. Schwabe and D. B. Graves, Phys. Rev. E 88, 023101 (2013).

[46] S. Plimpton, J. Comput. Phys. 117, 1 (1995).

[47] M. Schwabe, C.-R. Du, P. Huber, A. M. Lipaev, V. I. Molotkov, V. N. Naumkin, S. K. Zhdanov, D. I. Zhukhovitskii, V. E. Fortov, and H. M. Thomas, Microgravity Sci. Technol. 30, 581 (2018).

[48] K. Takahashi, Y. Hayashi, and S. Adachi, J. Appl. Phys. 110, 013307 (2011)

[49] A. V. Ivlev, G. E. Morfill, H. M. Thomas, C. Räth, G. Joyce, P. Huber, R. Kompaneets, V. E. Fortov, A. M. Lipaev, V. I. Molotkov et al., Phys. Rev. Lett. 100, 095003 (2008).

[50] A. M. Tichler, L. R. Gómez, N. Upadhyaya, X. Campman, V. F. Nesterenko, and V. Vitelli, Phys. Rev. Lett. 111, 048001 (2013). 